\title{
Non-linear Microwave Impedance of short and long Josephson Junctions
}

\author{
Z. Zhai, Patanjali V. Parimi, and S. Sridhar \\ Physics Department, Northeastern University, 360 Huntington Avenue, Boston, \\ MA 02115
}

(October 13, 2018)

\begin{abstract}
The non-linear dependence on applied $a c$ field $\left(b_{\omega}\right)$ or current $\left(i_{\omega}\right)$ of the microwave (ac) impedance $R_{\omega}+i X_{\omega}$ of both short and long Josephson junctions is calculated under a variety of excitation conditions. The dependence on the junction width is studied, for both field symmetric (current anti-symmetric) and field anti-symmetric (current symmetric) excitation configurations. The resistance shows step-like features every time a fluxon (soliton) enters the junction, with a corresponding phase slip seen in the reactance. For finite widths the interference of fluxons leads to some interesting effects which are described. Many of these calculated results are observed in microwave impedance measurements on intrinsic and fabricated Josephson junctions in the high temperature superconductors, and new effects are suggested. When a $d c$ field $\left(b_{d c}\right)$ or current $\left(i_{d c}\right)$ is applied, interesting phase locking effects are observed in the ac impedance $Z_{\omega}$. In particular an almost periodic dependence on the dc bias is seen similar to that observed in microwave experiments at very low dc field bias. These results are generic to all systems with a $\cos (\phi)$ potential in the overdamped limit and subjected to an ac drive.
\end{abstract}

\section{INTRODUCTION}

A Josephson junction (JJ) displays several remarkable properties which are well known [1.21. In the context of high temperature superconductors (HTS) the analysis of JJ has received new impetus for several reasons. In addition to the interest in fabricating barrier junctions made of HTS, there are new features observed in the electromagnetic response of the HTS materials which can be attributed to the occurrence of JJ distributed in the material. Many of the microwave properties of ceramics are ascribed to the presence of so-called "weak links" whose response is JJ-like. Josephson junction phenomena have been observed and attributed to Josephson coupling along the c-axis between superconducting layers [3], and even within the ab-plane [4].

Much of the analysis of an elemental JJ has been motivated by quantities which are experimentally accessible. The best studied parameters are the zero-frequency or $d c \mathrm{I}-\mathrm{V}$ characteristics, including the Shapiro steps seen in these $d c$ properties when the junction is irradiated by microwaves. These analyses have been extended to study Josephson junction arrays [5] which can be fabricated artificially using low $T_{c}$ materials, and are relevant in c-axis studies.

Recently, the high frequency or microwave impedance of single crystals has shown a remarkable non-linear dependence on the probing microwave field which can be described in terms of Josephson-like response to purely $a b$-plane currents [- [4]. Results such as these in single crystals, and in fabricated bicrystal thin film junctions [6], has resulted in the observation of new parameters, such as the dynamic or ac (microwave) resistance and reactance. These quantities were not addressed extensively previously because they were not of experimental interest until recently. It is therefore timely to carry out a detailed study of the high frequency properties of JJ, and the results of such a study are reported in this paper.

In this paper we present numerical simulations of the microwave impedance of short and long Josephson junctions under a variety of excitations or drive conditions. In the case of a short junction, the only excitation possible is an $a c$ current drive. For a long junction it is possible to study symmetric (the excitation field direction is the same on both sides of the junction), and antisymmetric (the excitation field direction is opposite on both sides of the junction) field excitations, and we consider both. We also examine the response of short and long junctions in the presence of an applied $d c$ field and, in the case of a long junction, the dependence of the impedance $Z_{\omega}$ on junction width $d$. These conditions are experimentally relevant and have not been analyzed in detail before. Some of the calculated results are consistent with experiments on crystals and films of HTS, while other new interesting effects are described which may be observable in future experiments.

\section{NONLINEAR IMPEDANCE OF SHORT JUNCTION}

The usual resistivitely-shunted JJ (RSJ) model typically applies to the short junction, in which the junction width is smaller than the Josephson penetration depth $\lambda_{J}$. In this limit the flux in the junction is uniformly distributed. 


\section{A. ac current driven RSJ}

Recently several authors have calculated the non-linear microwave impedance of a short RSJ [7] 10 , subjected to a pure $a c$ drive current represented as $I_{o} \sin (\omega t)$. In this model the Josephson junction is described by an ideal junction $\mathrm{J}$ shunted by a resistance $\mathrm{R}$ and capacitance $\mathrm{C}$ to form a parallel circuit. The total current $I$, flowing through the circuit has contributions from supercurrent $I_{S}$, normal current $I_{N}$ and displacement current $I_{D}$. While the normal current is caused by the flow of normal electrons in the barrier while the displacement current is due to the time varying electric field across the junction. Therefore, for the total current $I$ one can write

$$
I=I_{S}+I_{N}+I_{D}=I_{c} \sin (\phi)+\frac{\Phi_{0}}{2 \pi R} \frac{d}{d t} \phi+\frac{\Phi_{0} C}{2 \pi} \frac{d^{2}}{d t^{2}} \phi
$$

where, $\phi$ is the junction phase and $I_{c}$ is the critical current of the junction. $\Phi_{0}$ is the unit of a flux quantum, and the junction plasma frequency, $\omega_{p}$ is given by $\sqrt{2 \pi I_{c} / \Phi_{0} C}$. For low frequency drive currents $\left(\omega<<\omega_{p} \sim 100 G H z\right)$ the above equation can be written as,

$$
\beta^{-1} d \phi / d t+\sin (\phi)=i_{\omega} \sin (\omega t)
$$

Here, $\beta=2 e I_{c} R / \hbar, R$ is the shunt resistance and, $i_{\omega}=I_{o} / I_{c}$. It is convenient to do the analysis in dimensionless form

$$
d \phi / d \tau+\sin (\phi)=i_{\omega} \sin (\Omega \tau)
$$

where $\tau=\beta t$, and $\Omega=\omega / \beta$. The nonlinearity arises from the $\sin (\phi)$ term in Eq. 1. Eq. 1 is solved numerically for $\phi$. The $a c$ resistance, $R_{\omega}$ and reactance $X_{\omega}$ are calculated from the relation [4]

$$
Z_{\omega}=R_{\omega}+j X_{\omega}=\left(\frac{\omega}{2 \pi i_{\omega}}\right) \int_{0}^{2 \pi / \omega} \dot{\phi} e^{j \omega t} d t
$$

Fig. 1, shows the normalized $a c$ resistance and reactance versus microwave drive current $i_{\omega}$ for different $\Omega=$ $0.01,0.1,1$. As can be seen from the Fig. 1(a), when $\Omega$ is small, the $a c$ resistance shows several characteristic features: a threshold field and a subsequent parabolic rise, above the threshold, with step-like structures. The threshold current where a sudden increase in $R_{\omega}$ starts is defined as critical current $i_{\omega c}$. The step height decreases with increasing $i_{\omega}$. The reactance directly shows dynamic phase slips occurring as the drive current is increased(Fig. 1(b)). The sharp steps occur at values of $i_{\omega}$ for which there are bifurcations in the solution Eq. 3, i.e. $2 \pi$ phase slips in the gaugeinvariant phase difference $\phi$ across the junction. These bifurcations can cause sudden changes in $\mathrm{V}(\mathrm{t})$ during one cycle of the alternating current and hence result in steps [11]. When $\Omega$ becomes large, both the threshold and steps in $\mathrm{R}_{\omega}$ disappear, and the amplitude of the phase slip in $X_{\omega}$ becomes larger. Also, the phase slip moves to larger values of $i_{\omega}$ and the spacing between bifurcations becomes larger which results in $R_{\omega}$ and $X_{\omega}$ approaching $R$ (Fig. 1).

\section{NONLINEAR IMPEDANCE OF LONG JUNCTION}

A Josephson junction is considered long when the junction width is larger than the Josephson penetration depth, $\lambda_{J}$. In this case, for applied magnetic field more than the junction critical field, $H_{c 1 J}=\left(\Phi_{0} / 4 \pi \lambda_{J}^{2}\right) \ln \left(\lambda_{J} / t_{i}\right)$ [12, 13 , the field enters the junction in the form of Josephson fluxons. $\lambda_{J}=\sqrt{\Phi_{0} /\left[2 \pi \mu_{o} J_{o}\left(2 \lambda_{L}+t_{i}\right)\right]}$ is the Josephson penetration depth, and $\lambda_{L}$ is the London penetration depth. The RSJ model, therefore, cannot give an adequate description of the ac or dc field responses. Fig. 2 gives a schematic representation of a long junction for both symmetric excitation, when the microwave field $b_{\omega}$ is applied symmetrically on either side of the junction, in which case the induced current is anti-symmetric, and antisymmetric excitation due to the ac field $b_{\omega}$, whence the microwave drive current flows symmetrically through the junction.

The relation between the phase difference across the junction and the magnetic field along the junction is given by

$B_{z}(x, t)=-\frac{\Phi_{0}}{2 \pi\left(2 \lambda_{L}+t_{i}\right)} \frac{\partial \phi(x, t)}{\partial x}$, the relation between $\phi$ and electric field across the junction is $E_{y}(x, t)=\frac{\Phi_{0}}{2 \pi t_{i}} \frac{\partial}{\partial t} \phi(x, t)$ and the current density is $J_{y}(x, t)=J_{c} \sin \phi+E_{y} / \rho_{i}$. Here, the first term represents supercurrent density and the second term normal leakage current density, $J_{y}(x, t)$ is related to $B_{z}$ and $E_{y}$ by Ampere's law with a displacement current. By combining the above relations, for frequencies smaller than the plasma frequency, the model that represents the junction can be written as [8]. 


$$
\lambda_{J}^{2} \frac{\partial^{2} \phi}{\partial x^{2}}-\beta^{-1} \frac{\partial \phi}{\partial t}=\sin \phi
$$

$\beta=2 \pi \rho_{i} t_{i} J_{c} / \phi_{o}$ is a damping parameter. Here, $t_{i}$ is the junction thickness, $\rho_{i}$ and $\epsilon$ are resistivity and permittivity of the junction material, $J_{c}$ is the critical current density of the junction, and $\phi_{o}$ is the flux quantum. In dimensionless form the equation is written as

$$
\frac{\partial^{2} \phi}{\partial X^{2}}-\frac{\partial \phi}{\partial \tau}=\sin \phi
$$

where $X=x / \lambda_{J}$, and $\tau=\beta t$.

The detailed study of the long junction depends on the parameter values and on the boundary conditions, which will be introduced in the following for the particuler problem considered. When the the applied microwave field $B_{\omega}=B_{o} \sin \omega t$ is along $\widehat{z}$-axis and junction width infinite, the boundary conditions at $x=0$ and $x=d$ are

$$
\left[\frac{\partial \phi}{\partial x}\right]_{x=0}=-b_{\omega} \sin (\Omega \tau),\left[\frac{\partial}{\partial x} \phi\right]_{x=d \rightarrow \infty}=0 .
$$

Where, $b_{\omega}=2 \pi B_{o}\left(2 \lambda_{L}+t_{i}\right) / \phi_{o}, \Omega=\omega / \beta$. Eq. 7 is solved numerically for $\phi$ and, as in the case of small junction. Using Eq. 4 and boundary conditions the expressions for $R_{\omega}$ and $X_{\omega}$ are obtained.

Fig. 3 shows $R_{\omega} / R_{n}$ and $X_{\omega} / R_{n}$ versus $b_{\omega}$ for different values of $\Omega=0.01,0.1$ and 1 , for an infinite long junction with $R_{n}=\sqrt{\mu_{o} \rho_{i} \omega\left(2 \lambda_{L}+t_{i}\right) /\left(2 t_{i}\right)}$. When $\Omega$ is small, the variation in $R_{\omega}$ is similar to the RSJ model calculation, in that it has a threshold and a parabolic rise with step-like structures. However, no clear phase slips are observed in $X_{\omega}$ versus $b_{\omega}$. When $\Omega$ is large, at the threshold the jump becomes higher and for further increase in $b_{\omega}$ the steps become smooth. When $\Omega$ is very large the steps are washed out. The $a c$ reactance $X_{\omega}$ also shows a similar transitions from a step-like response to a smooth curve.Indeed the overall behavior of $R_{\omega}$ and $X_{\omega}$ are similar, this is essentially due to the "bulk" nature of the configuration. In the long junction the ac impedance is associated with the vortex - antivortex propagations. For short junctions the notion of a vortex - antivortex pair does not appear because the Josephson vortex has a finite size of the order $2 J_{c} \lambda_{J}$. Therefore, the physical mechanism leading to step structures in short and long junctions are different. For long junctions the steps are due to synchronous coupling of the oscillating vortex or antivortex with the ac current [14]. Essentially, the steps in Fig. 3 occur due to the changes in the number of vortices in the junction and the step height is dependent on the microwave power. For higher values of $\Omega$ vortex entry takes place at higher field and hence steps become broader and are moved to larger values of $b_{\omega}$.

In the limit the junction width $d \sim \lambda_{J}$, the boundary condition $\left[\frac{\partial \phi}{\partial x}\right]_{x=d} \neq 0$. This problem can be studied in two ways, the symmetric and antisymmetric cases (see Fig. 2).

\section{A. Symmetric field excitation (antisymmetric current drive)}

When a microwave field is applied on either side of the junction ( Fig. 2a), then this corresponds to a symmetric microwave excitation. The boundary condition in this case is

$$
\left[\frac{\partial}{\partial x} \phi\right]_{x=0, d}=-b_{\omega} \sin (\Omega \tau)
$$

In Fig. $4 R_{\omega} / R_{n}$ versus $b_{\omega}$ responses for three different values of $\Omega$ and four different values of the width, $d / \lambda_{J}=5$, 10,50 and $\infty$, are shown. When $\Omega$ is small (Fig.4a), $R_{\omega}$ is small for low values of $d / \lambda_{J}$ compared to the high values of the latter. $R_{\omega}$ approaches $R$ at high values of $b_{\omega}$ for infinite junction width. The threshold and the steps can be seen clearly for all values of $d / \lambda_{J}$. On the contrary, when $\Omega$ is large (Fig. 4c), it can be seen that there is almost no effect on $R_{\omega}$ with the change in the junction width, except when junction width is too small.

Both at high field $\left(b_{\omega} \rightarrow \infty\right)$ and large damping $(\Omega \sim 1)$ the junction behaves like a normal metal with $R_{\omega}$ approaching its asymptotic value $R_{n}$. The result can be understood by considering the fact that at these two conditions, the $R_{\omega}$ response is dominated by loss arising from normal current than the supercurrent. The manifestation of dominance of normal contribution can be seen in Fig. 4(c) in two ways. Firstly, the $R_{\omega}$ response at larger damping is smooth sans any threshold and step like features. Secondly, since the normal current contribution to $R_{\omega}$ depends 
on the Josephson skin depth, which is invariant for any fixed $\Omega$, variation in the junction width does not have much affect on $R_{\omega}$, and therefore, results in the same change in the magnitude of $R_{\omega}$ at various $d / \lambda_{J}$ values.

However, for all the values of $\Omega$ when $d / \lambda_{J}$ is too small $\left(d / \lambda_{J}=2\right)$, it can be seen that $R_{\omega}$ is small. In fact, as $d / \lambda_{J}$ approaches zero, the junction effects should not be observed as the total current gets canceled out due to asymmetry on either sides of the junction.

\section{B. Antisymmetric field excitation (symmetric current drive)}

If an $a c$ current is passed through the junction ( Fig. 2b), then the direction of the induced magnetic field is opposite on both sides of the junction making this configuration an antisymmetric field-driven case. The corresponding boundary conditions are

$$
\left[\frac{\partial}{\partial x} \phi\right]_{x=0}=-b_{\omega} \sin (\Omega \tau),\left[\frac{\partial}{\partial x} \phi\right]_{x=d}=b_{\omega} \sin (\Omega \tau)
$$

In Fig. 5 plots of $R_{\omega} / R_{n}$ versus $b_{\omega}$ for $d / \lambda_{J}=5,10,20$ and $\infty$, at three different values of $\Omega$, are shown. When $\Omega$ is small, compared to the infinite width case (Fig. 3), $R_{\omega}$ is larger for small widths. It is worth noting here that this response is quite opposite to the symmetric field excitation, discussed in the earlier section, where $R_{\omega}$ is larger when the width is large. When $\Omega$ is large enough $(\Omega=1)$, as in the symmetric case, the change in width does not show much affect on $R_{\omega}$, unless the width is too small. The interesting case, here, is when $\Omega$ is in the middle region $(\Omega=0.1$, Fig. $5 \mathrm{~b})$. In this case for junction width several times the Josephson penetration $\operatorname{depth}\left(d / \lambda_{J}=5\right)$, the $R_{\omega}$ response shows a threshold and rises parabolically with the step-like structures for increasing $b_{\omega}$. It is similar to the result of short junction RSJ calculation and long junction small $\Omega$ case. As the junction width increases a few times more $\left(d / \lambda_{J}=10\right), R_{\omega}$ shows sudden jumps as $b_{\omega}$ increases. This jumpiness disappears for higher values of junction width, and eventually, when $d / \lambda_{J}=\infty$ the steps become broader and smooth.

These results can be explained by invoking the fact that in the antisymmetric field excitation since the currents on both sides of the junction flow in the same direction, as $d / \lambda_{J}$ decreases current density increases, for any particular field. Such a current crowding causes $R_{\omega}$ to increase for smaller values of $d / \lambda_{J}$. Also, the difference between the step patterns in the symmetric and antisymmetric field excitations is due to different vortex or antivortex interactions with ac current, as they move across the junction width.

\section{NONLINEAR IMPEDANCE OF DC BIASED (CURRENT OR FIELD) JUNCTION}

An important class of experiments is where the microwave absorption is measured in the presence of a dc magnetic field which is varied. For this reason, we consider below the case of a JJ which is biased by a dc current or magnetic field. Naturally, the short junction is biased by a dc current, and the infinitely long junction by a dc magnetic field ( we consider the case of symmetric excitation only), and we calculate the ac impedance as a function of the dc bias.

\section{A. Short junction : dc current biased, ac current driven}

If a dc current, $I_{d c}$ is superposed on $i_{\omega}$, the driving current becomes $I_{o} \sin (\omega t)+I_{d c}$. The corresponding junction equation can then be written as

$$
d \phi / d \tau+\sin (\phi)=i_{\omega} \sin (\Omega \tau)+i_{d c}
$$

where $i_{d c}=I_{d c} / I_{c}$. In Fig. 6, we plot $R_{\omega}$ and $X_{\omega}$ versus $i_{\omega}$ for various values of $i_{d c}=0,0.5,2$, and 10 at fixed $\Omega=0.1$. There are two regimes of behavior noticeable, corresponding to $i_{d c}>i_{\omega c}$ or $i_{d c}<i_{\omega c}$.

When $i_{d c}=0$, the critical current $i_{\omega c}\left(i_{d c}=0\right)$ is found to be 1.1 (Fig. 6). When the $d c$ drive current $i_{d c}$ is smaller than the zero $d c$ critical current (i.e. $0<i_{d c}<1.1$ ), compared to the response of $R_{\omega}$ versus $i_{\omega}$ for $i_{d c}=0$, it can be seen that the critical current $i_{\omega c}$ decreases and that both the step size and step rising pattern are changed. In Fig. 7 , we plot the critical current $i_{\omega c}$ as a function of $i_{d c}$ for $\Omega=0.1$. A linear fit $i_{\omega c}=a i_{d c}+b$ yields $a \approx-1$ and $b \approx 1.1$. Note however that this critical current value is weakly frequency-dependent.

When $i_{d c}$ is larger than the zero $d c$ critical current (i.e. $i_{d c}>1.1$ ), initially apparent jumps appear and with further increase of the $a c$ drive current $i_{\omega}, R_{\omega}$ begins to decrease with sudden intermittent steps and eventually approaches 
the $R_{\omega}\left(i_{\omega} \rightarrow \infty, i_{d c}=0\right)$. This regime $i_{d c}, i_{\omega}>i_{\omega c}$ is very interesting as it appears to show strong phase-locking effects in the ac impedance, similar to the Shapiro steps seen in dc $I-V$ characteristics.

For very large values of $i_{d c}\left(i_{d c}=10\right)$, as expected, the effect of the microwave current is negligible and the $a c$ resistance approaches the shunt resistance $R$.

In the case of $a c$ reactance $X_{\omega}$, which is plotted in Fig. 6 bottom panel, the phase slip is not affected by the increase in the $d c$ current $i_{d c}$, however, the amplitude of phase slip reduces dramatically for larger values of $i_{d c}$.

In Fig. 8, we plot $R_{\omega}$ and $X_{\omega}$ versus $i_{d c}$ for different microwave drive currents $i_{\omega}=0.5,1,2,10$. It can be observed from the figure that for all values of $i_{\omega}$, as $i_{d c}$ increases, $R_{\omega}$ initially increases, reaches a maximum and approaches $R$ eventually. On the other hand, $\mathrm{X}_{\omega}$ decreases initially with the increase in $i_{d c}$. The occurrence of phase slip can be seen clearly when $i_{d c}$ is small. Large values of either $i_{\omega}$ or $i_{d c}$ diminishes the effect of phase slip.

\section{B. Long Junction : dc magnetic field biased, ac field driven}

The presence of dc magnetic field, $B_{d c}$ is studied for infinite long junction because in this case the $R_{\omega}$ response is same in both symmetric and antisymmetric excitations, as can be seen in Figs. 4 and 5 . The calculations are, however, done for symmetric excitaion. When $B_{d c}$ is applied in addition to the microwave field, the boundary condition becomes

$$
\left[\frac{\partial}{\partial x} \phi\right]_{x=0}=-b_{\omega} \sin (\omega t)-b_{d c} .
$$

where, $b_{d c}=2 \pi B_{d c}\left(2 \lambda_{L}+t_{i}\right) / \phi_{o}$. Considering $\Omega=0.1$ and $d / \lambda_{J}=\infty$ case, the $R_{\omega} / R_{n}$ versus $b_{\omega}$ response at various $b_{d c}=0,0.1,1$ are shown in Fig. 9. In Fig. 10 the plots of $R_{\omega} / R_{n}$ versus $b_{d c}$ for two different $b_{\omega}$ values, $b_{\omega}=0.1$ and 1 , are shown. It can be seen in Fig. 9 that when the dc field is high, $R_{\omega}$ at low ac field is high and as $b_{\omega}$ increses it decreases and reaches the asymptotic value $R_{\omega}$ when $b_{\omega} \rightarrow b_{d c}$. This high ac resistance is due to the loss arising from vortex flow whose response is dependent on the intrinsic pinning in the junction. The same mechanism results in $R_{\omega}$ increasing beyond $R_{n}$ as the dc field is increased.

A very intriguing result is the case for intermediate $b_{\omega}=0.1$, shown in Fig. 10, for the parameter $\Omega=0.1$. The field dependence is strikingly regular, almost periodic. As noted below, this is similar to experimental data seen in microwave absorption experiments on YBCO single crystals.

\section{REMARKS}

The recent observation that bulk single crystals of high temperature superconductors behave like Josephson junctions in their microwave response, and are even of higher quality than fabricated junction [4], was the original motivation for this work. In Fig. 11 the experimental microwave surface resistance variation as a function of microwave field $\left(\mathrm{H}_{\mathrm{rf}}\right)$ recorded on a high quality single crystal of $Y \mathrm{Ba}_{2} \mathrm{Cu}_{3} \mathrm{O}_{7-\delta}$, at temperature of $3 \mathrm{~K}$, is shown. It is important to note that the $R_{s}$ variation mimics a single long junction response for symmetric excitation (see Fig.3), with $\Omega=0.03$. Since the measuring frequency is $10 \mathrm{GHz}$, this gives a damping frequency of $330 \mathrm{GHz}$ equivalent to an $I_{c} R=0.8 \mathrm{meV}$. A detailed comparison of experimental data with simulations will be presented elsewhere.

Very interesting results which can be attributed to JJ's have been observed in BSCCO [15, 16]. There the microwave surface resistance shows sudden jumps or transitions between apparently quantized values when the microwave field $H_{\omega} \| \hat{c}$-axis. However, it is probably necessary here to also include the inertial term even in the RSJ model, which leads to a very rich dynamics and apparently chaotic behavior.

Another class of experiments which have been analyzed using an RSJ model is the Modulated Microwave Absorption experiments [17, in which the microwave absorption is measured in the presence of a dc bias field, along with a very low frequency $(<100 \mathrm{~Hz})$ modulation field. Here again the properties calculated were in terms of dc quantities, and not the true microwave impedance as is done here, and hence the analysis should be redone along the lines of the present paper, as we have in the previous section.

Indeed, as Fig. 10 shows, a striking nearly periodic behavior on the microwave bias field can be observed in the caculations. Similar periodic behavior has often been observed in modulation experiments on single crystals [18]. Our calculations shows that this type of behavior can occur in long junctions for a judicious choice of the parameters $\Omega$ and $b_{\omega}$.

A surprising theme that emerges from the experiments mentioned above, when compared with calculations as presented in this paper, is that the experimental data can often be well described by the response of a single junction, rather than the multitude of "weak links" one would expect due to inhomogeneities. Thus the experiments and analysis taken together strongly suggest the presence of a single periodic $(\sin (\phi))$ response of the superconductor to 
applied magnetic fields. One can imagine that this arises from a single dominant weak link in the system, or of many weak links acting coherently. As pointed out in ref. [4, the degree of coherence is striking and strongly suggests a fundamental mechanism, such as the possibility of bulk coupled superconductors.

Previously, several authors have modeled Josephson junction response in granular superconductors in terms of strongly coupled and weakly coupled Josephson junctions. Experimentally, it is found that HTS of different families often respond to microwave excitations in a similar manner, including ceramics and films and different materials like YBCO and BSCCO. Hylton et al. [19] have used a resistively shunted junction (RSJ) model to describe the residual microwave losses in thin films. In this model the grains are considered to be purely inductive and the grain boundary weak links the RSJ's. This model has later been expanded and used by various authors [20 22]. However it is now clear that this early analysis needs to be replaced by the more exact analysis such as presented in this paper and by McDonald et. al. [8].

While the discussion in this paper has been entirely in the context of superconducting Josephson junctions, we are aware that the fundamental equations are applicable to a variety of nonlinear systems. These results are generic to all systems with a $\cos (\phi)$ potential in the overdamped limit subjected to an ac drive. Hence the results presented here have a general validity beyond superconductivity, wherever the ac impedance is a significant quantity which is experimentally accessible. A particularly interesting system is that of striped metals, where the dynamical response is again of the Josephson type [23], and where all the analysis obtained here should apply .

In summary, we have presented the nonlinear ac resistance and reactance analysis for both short and long junctions under various conditions. In the case of a short junction, the higher damping leads to less pronounced nonlinearities. The steps observed in the $a c$ resistance are due to $2 \pi$ phase slips in the gauge invariant phase difference across the junction. The application of dc field reduces the critical current and when the dc current is more than the ac current, phase locking effects are seen in the ac impedance, similar to Shapiro steps in the dc $I-V$ characteristics.

In the case of a long junction, the step-like features in $a c$ impedance appear due to changes in the number of fluxons. When the damping is large, the steps are pushed to higher values of current as the vortex entry takes place at high currents. In the symmetric and antisymmetric filed excitations it found that, at large damping or high fields, the $a c$ resistance response is dominated by the resistive (normal) contribution and hence leads to same magnitude and variation of $R_{\omega}$, for various values of the junction width. Overall, for both short and long junctions, in high current and large damping limit, the $a c$ resistance approaches the limiting dc resistance value and the nonlinearities in its response are less pronounced. These interesting features may be observable in experiments on crystals and films of HTS and other superconductors.

\section{ACKNOWLEDGMENTS}

Work at Northeastern University was supported by NSF-ECS-9711910.

[1] K. K. Likharev, Dynamics of Josephson junctions and circuits, Gordon and Breach Science Publishers, New York (1986).

[2] A. Barone and G. Paterno, Physics and Applications of Josephson effects, John Wiley and Sons (1982).

[3] R. Kleiner, and P. Müller, Phys. Rev. Lett., 68, 2394 (1992). R. Kleiner, and P. Müller, Phys. Rev. B 49, 1327 (1994).

[4] Z. Zhai, H. Srikanth, S. Sridhar, A. Erb, E. Walker, and R. Flukiger, Physica C 282-287, 1601 (1997).

[5] N. F. Pedersen, and A. Davidson, Phys. Rev. B 41, 178 (1990).

[6] D. E. Oates, P. P. Nguyen, Y. Habib, G. Dresselhaus, M. Dresselhaus. G. Koren, and E. Polturak, Appl. Phys. Lett. 68, 705 (1996)

[7] S.Sridhar, unpublished.

[8] J. McDonald, and John R. Clem, Phys. Rev. B 56, 14723 (1997).

[9] M.Hein, Studies of High Temperature Superconductors, Ed. A.Narlikar, Vol. 18, pp 141, Nova Science Publishers, New York (1996).

[10] L. M. Xie, J. Wosik, and J. C. Wolfe, Phys. Rev. B 54, 15494 (1996).

[11] In ref. [8], the authors analyse the sudden changes in $\mathrm{v}(\mathrm{t})$ for various values of $i_{\omega}$. It is found that for two different values of $i_{\omega}$ which are close by, for any particular $\tau$, the voltage responses differ from each other by a change in the sign of the voltage pulses. Therefore, the sharp features in $R_{\omega}$ and $X_{\omega}$ correspond to the inversion of a voltage pulse at two different times during one cycle of the alternating current.

[12] M. Tinkham, Introduction to Superconductivity, 2nd ed., McGraw-Hill, New York (1996). 
[13] J. R. Clem, Physica C 153-155, 50 (1988).

[14] Jhy-Juin Chang, Phy. Rev. B 34, 6137 (1986).

[15] T. Jacobs, Balam A. Willemsen, S. Sridhar, Qiang Li, G. D. Gu, and N. Koshizuka, World Scientific, pp 288 (1996).

[16] T. Jacobs, Balam A. Willemsen, and S. Sridhar, Rev. Sci. Instrum. 67, 3757 (1996).

[17] A. M. Portis, K. W. Blazey, K. A. Müller, and J. G. Bednorz, Europhys. Lett. 5, 467 (1988), S. V. Bhat, P. Ganguly, T. V. Ramakrishnan, and C. N. R. Rao, J. Phys. C 20, L559 (1987).

[18] A. Dulcic, B. Rakvin, and M. Pozek, Europhys. Lett. 10, 593 (1989), K. Kish, S. Tyagi, and C. Kraft, Phys. Rev. B 44, 225 (1991).

[19] T. L. Hylton, A. Kapitulnik, M. R. Beasley, John P. Carini, L. Drabeck, and George Grüner, Appl. Phys. Lett. 53, 1343 (1988).

[20] P. P. Nguyen, D. E. Oates, G. Dresselhaus, and M. S. Dresselhaus. Phys. Rev. B 48, 6400 (1993)

[21] M. Mahel, Solid State Commun. 97, 209 (1996).

[22] C. Attanasio, L. Maritato, and R. Vaglio, Phys. Rev. B 43, 6128 (1991)

[23] N. Hasselmann, A. H. Castro Neto, C. Morais-Smith, and Y. Dimashko, cond-mat/9807070 (preprint)

\section{FIGURE CAPTIONS}

FIG. 1. RSJ model calculation of ac $(a)$ resistance and $(b)$ reactance versus $i_{\omega}$ of a short junction for three different values of $\Omega$, the damping parameter. The step like features seen in $R_{\omega}$ are due to $2 \pi$ phase slips in the phase difference across the junction.

FIG. 2. Cross sectional view of long Josephson junction for $(a)$ symmetric field excitation - antisymmetric current drive, and $(b)$ antisymmetric field excitation - symmetric curret drive.

FIG. 3. (a) $R_{\omega}$ and (b) $X_{\omega}$ versus $i_{\omega}$ for infinite long junction for various values of $\Omega$. The step like structures are due to changes in the number of vortices in the junction. At higher values $\Omega$ vortex nucleation takes place at higher currents and hence the steps are pushed to higher current values.

FIG. 4. $\quad R_{\omega}$ versus $b_{\omega}$ for symmetric field excitation when $\Omega=0.01(a), 0.1(b)$, and $1.0(c)$, and for various junction widths. When the damping is high and for higher currents the resistive response dominates and hence threshold step like features disappear in the $R_{\omega}$ response (bottom panel).

FIG. 5. $\quad R_{\omega}$ versus $b_{\omega}$ for antisymmetric field excitation when $(a) \Omega=0.01,(b) 0.1$ and $(c)$ 1.0, and for varying junction widths.

FIG. 6. $R_{\omega}(a)$ and $X_{\omega}(b)$ versus $i_{\omega}$ of short junction in the presence a dc current $i_{d c}$. When $i_{d c}$ is smaller than the zero $d c$ critical current, $i_{\omega c}\left(\right.$ i.e. $\left.0<i_{d c}<1.1\right)$, it can be seen that $i_{c}$ decreases. When $i_{d c}$ is larger than the zero dc critical current (i.e. $\left.i_{d c}>1.1\right), R_{\omega}$ begins to decrease with sudden intermitent steps and eventually approaches the the limiting resistive value. This regime $i_{d c}, i_{\omega}>i_{\omega c}$ is very interesting as it appears to show phase-locking effects in the ac impedance, similar to the Shapiro steps seen in dc $I-V$ characteristics.

FIG. 7. Variation of $i_{\omega c}$ with $i_{d c}$. Note that $i_{\omega c}$ decreases linearly with slope $\approx-1$.

FIG. 8. (a) $R_{\omega}$ and (b) $X_{\omega}$ versus $i_{d c}$ for various values of $i_{\omega}$. With the increase in $i_{d c}$, while $R_{\omega}$ increases initially, $X_{\omega}$ decreases. The occurance of phase slip is pronounced at low ac currents.

FIG. 9. $R_{\omega}$ dependence on $b_{\omega}$ for an infinite long junction when $\Omega=0.1$ in the presence of $d c$ field. 
FIG. 10. $R_{\omega}$ dependence on $b_{d c}$ for an infinite long junction when $\Omega=0.1$ in the presence of $a c$ field.

FIG. 11. Microwave surface resistance $\left(R_{s}\right)$ versus microwave magnetic field $\left(H_{r f} \| c-\right.$ axis $)$ recorded at $10 G H z$ abd $3 K$, of a very high quality single of $\mathrm{YBa}_{2} \mathrm{Cu}_{3} \mathrm{O}_{7-\delta}$. Note that the $R_{s}$ variation can very well be described by a single Josphson junction response. 

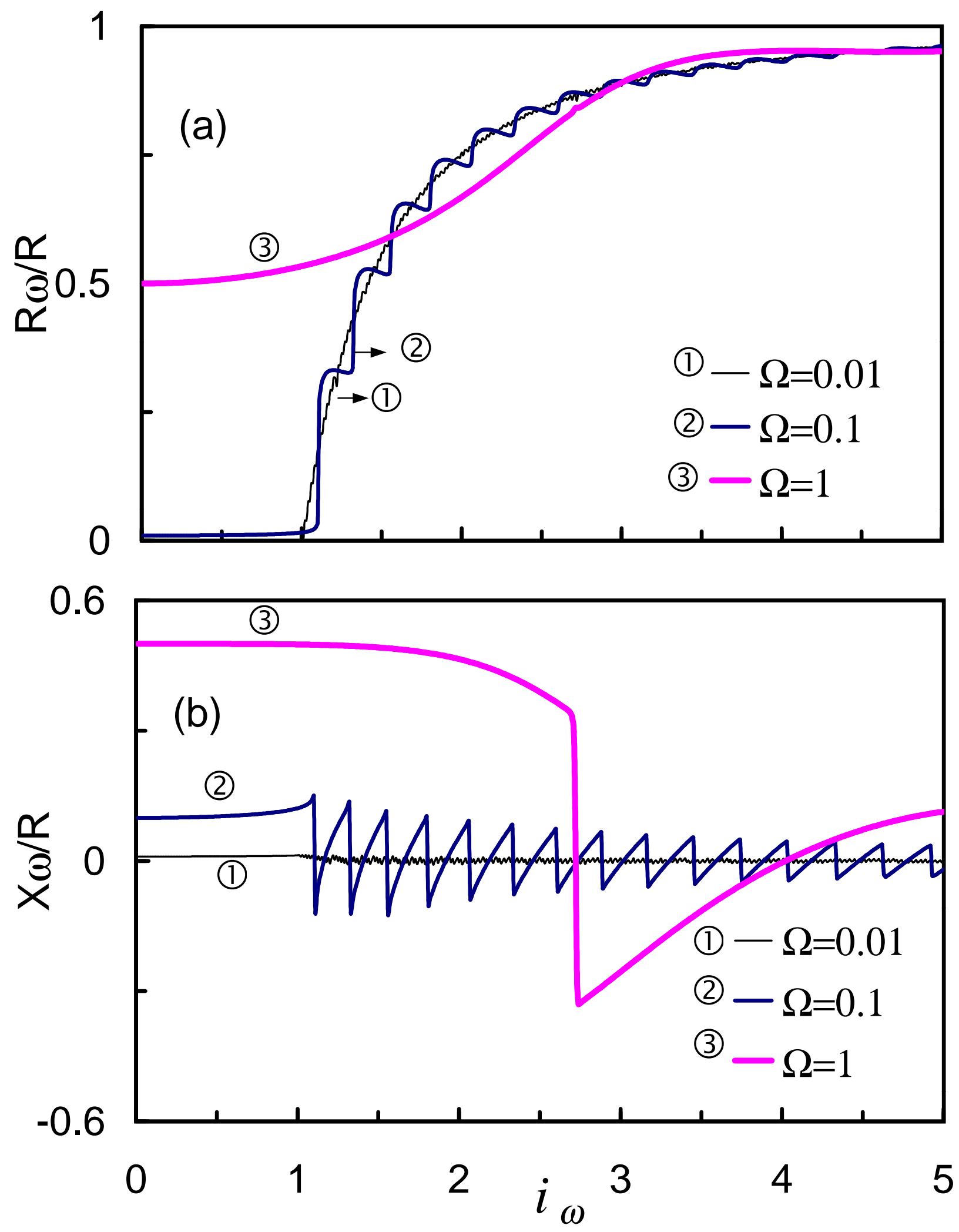


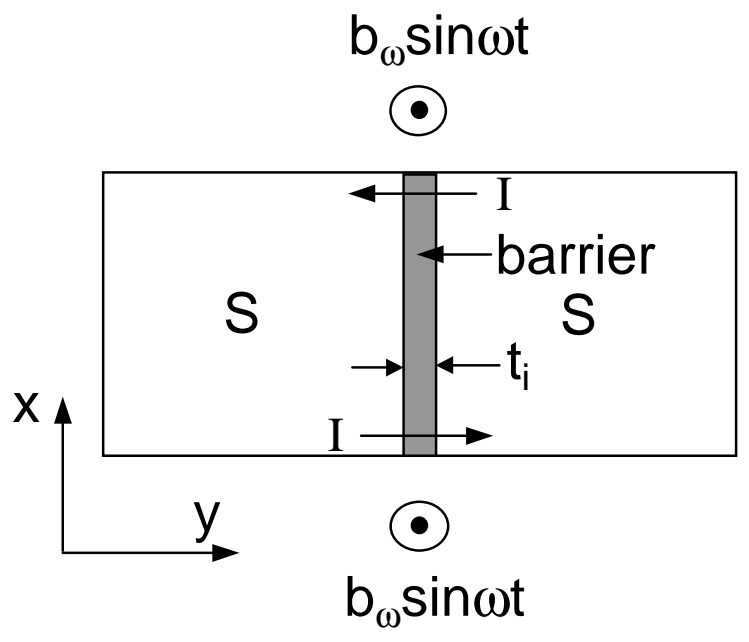

(a)

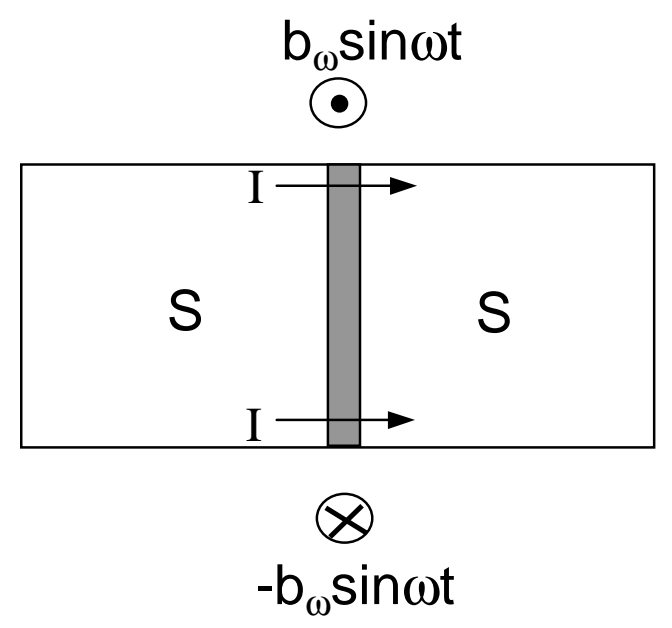

(b) 

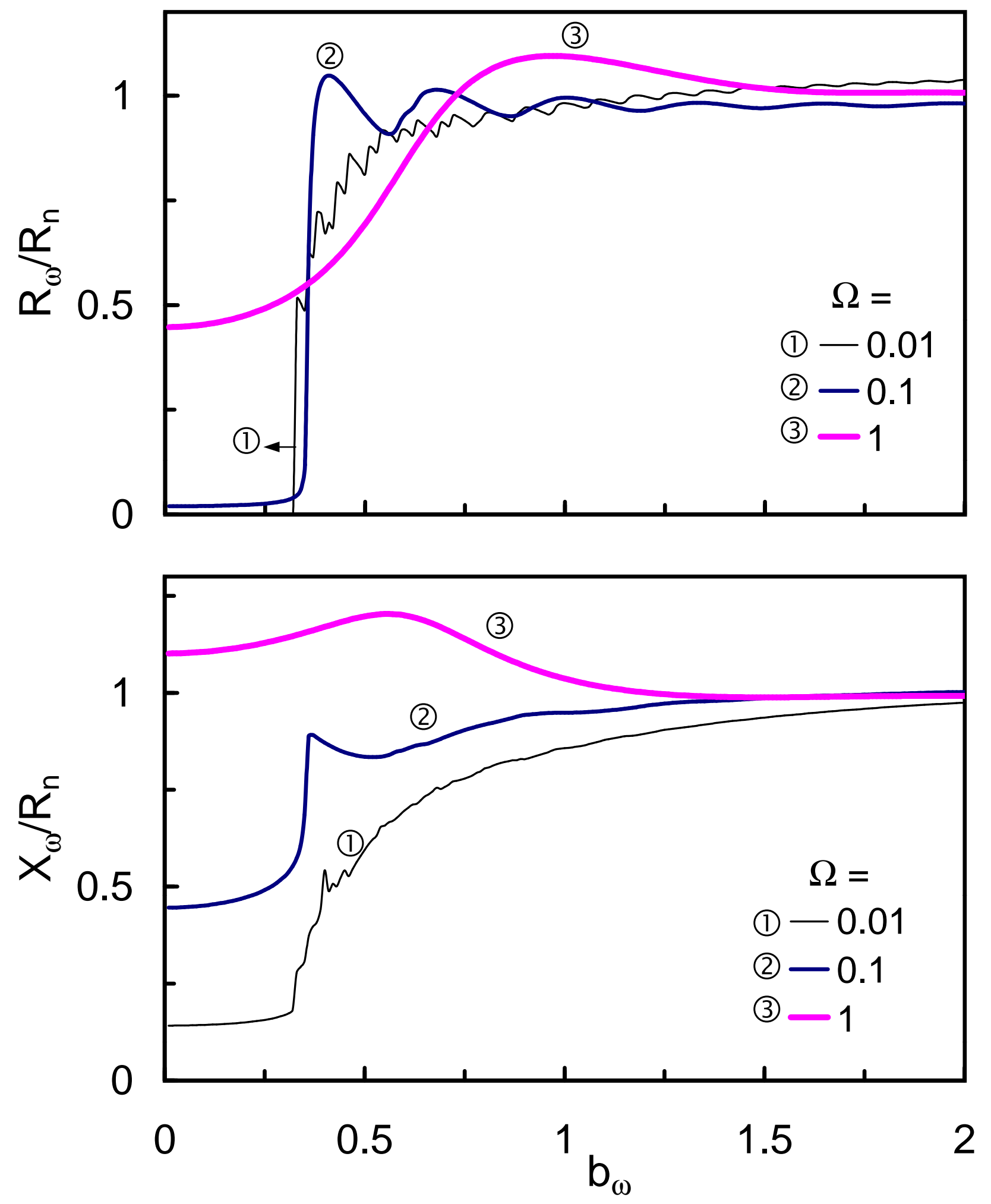

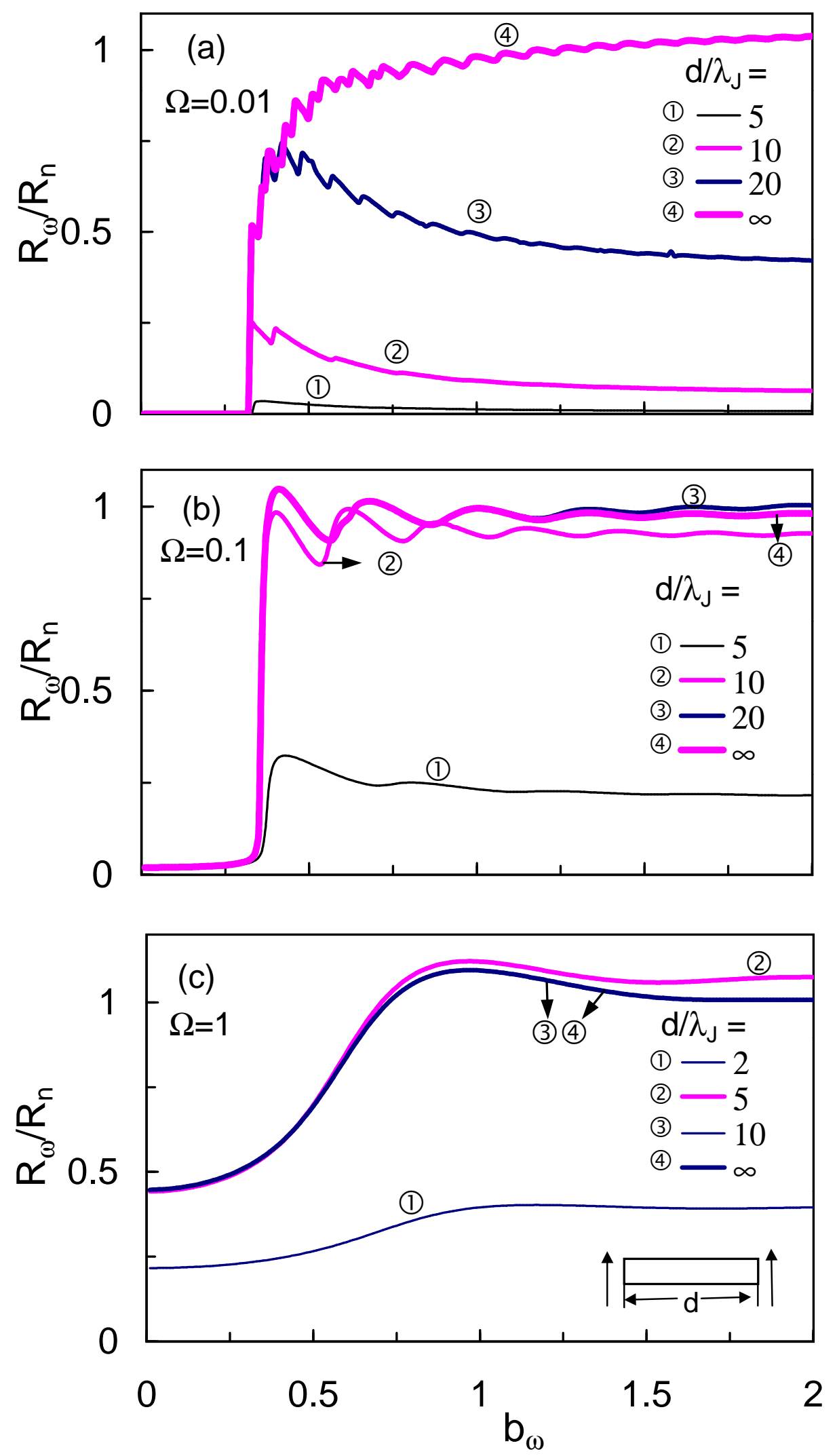

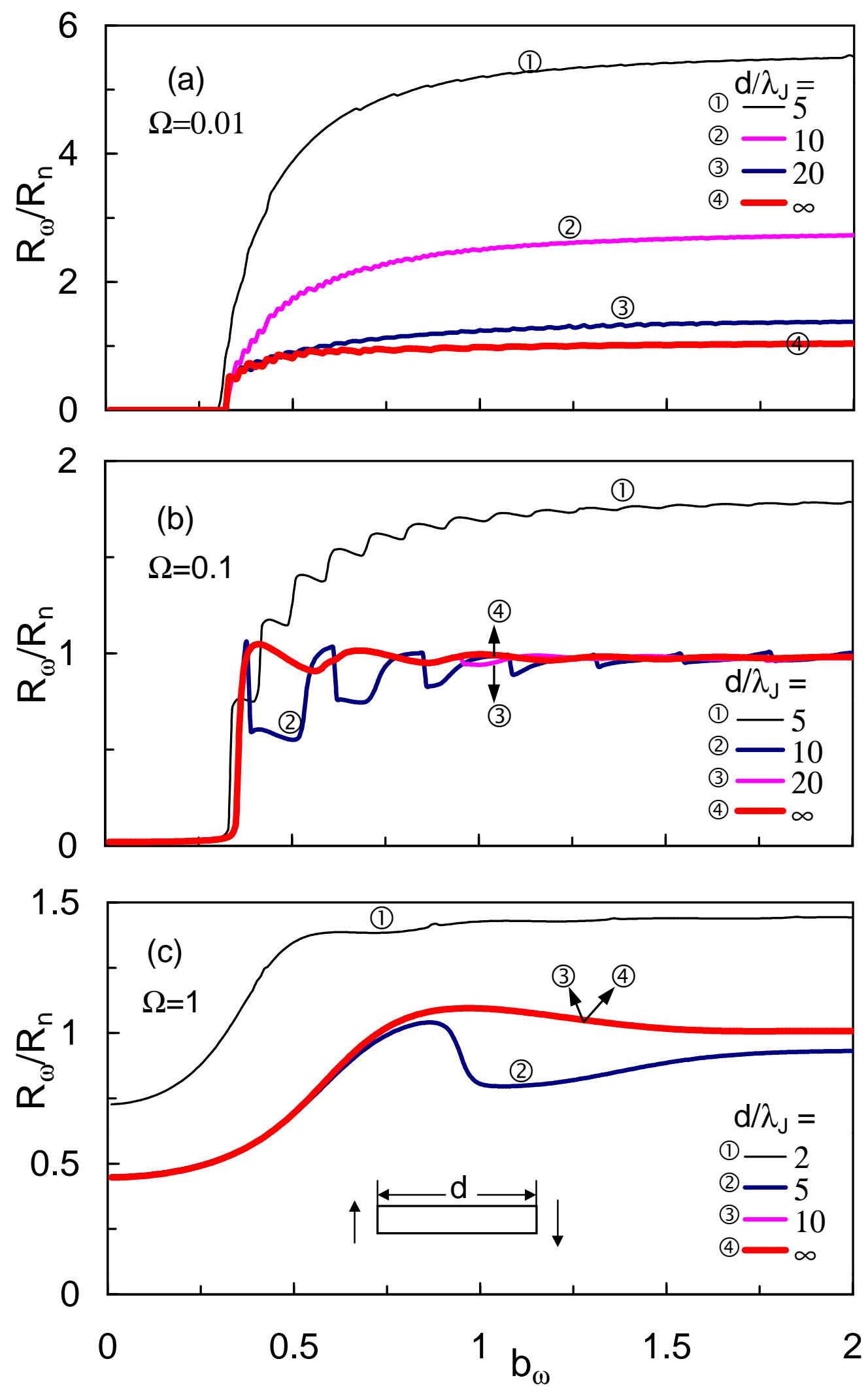

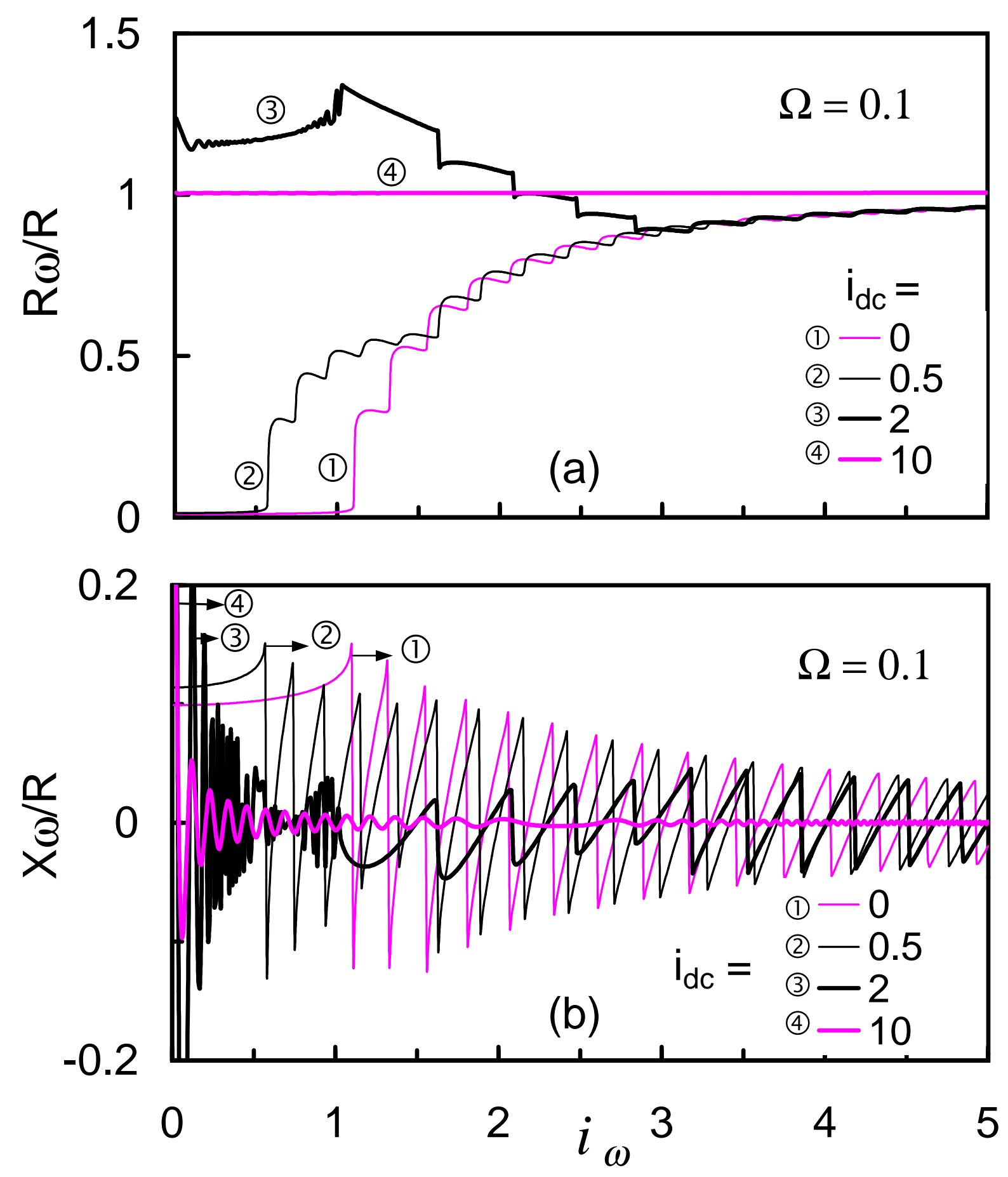


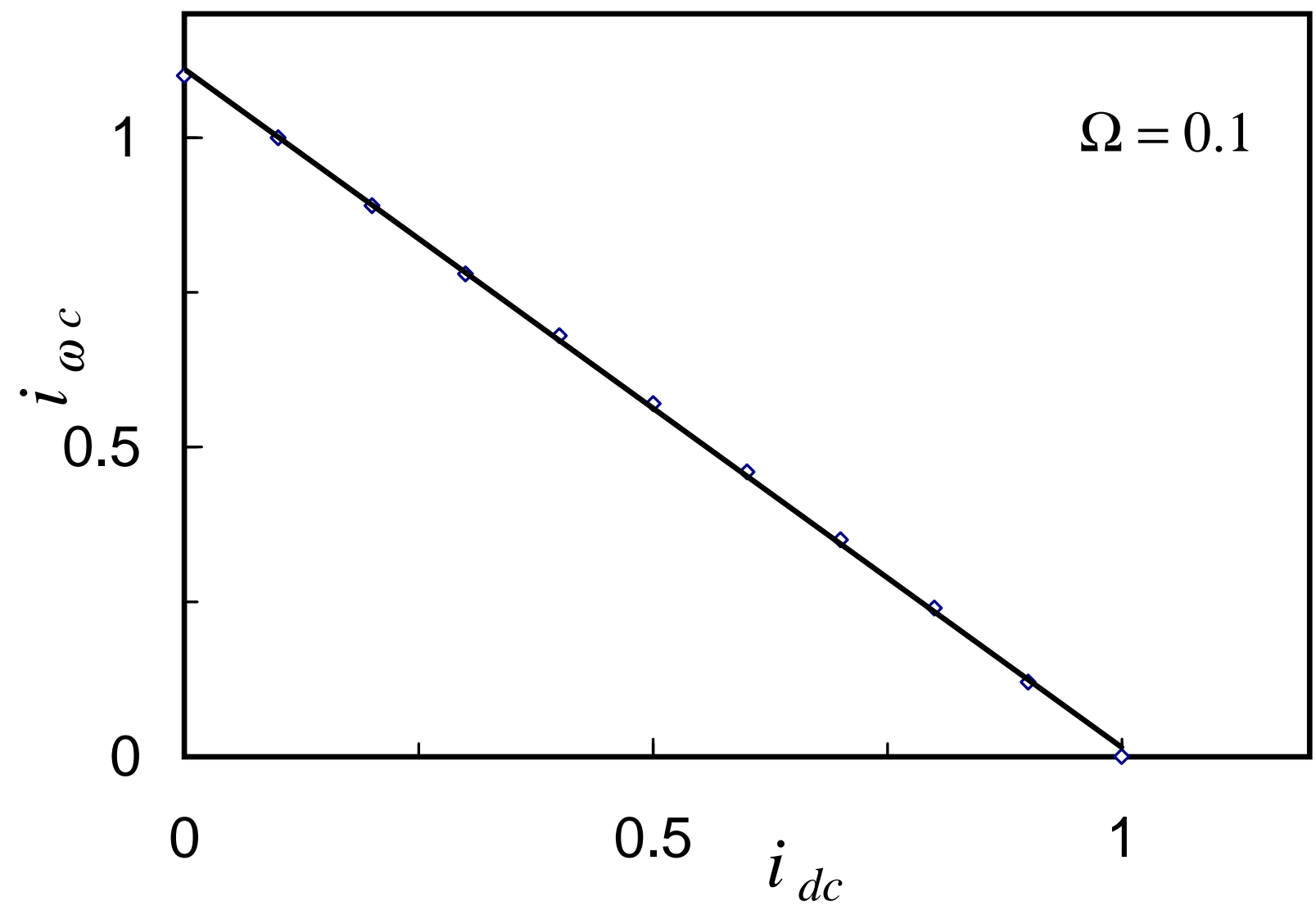



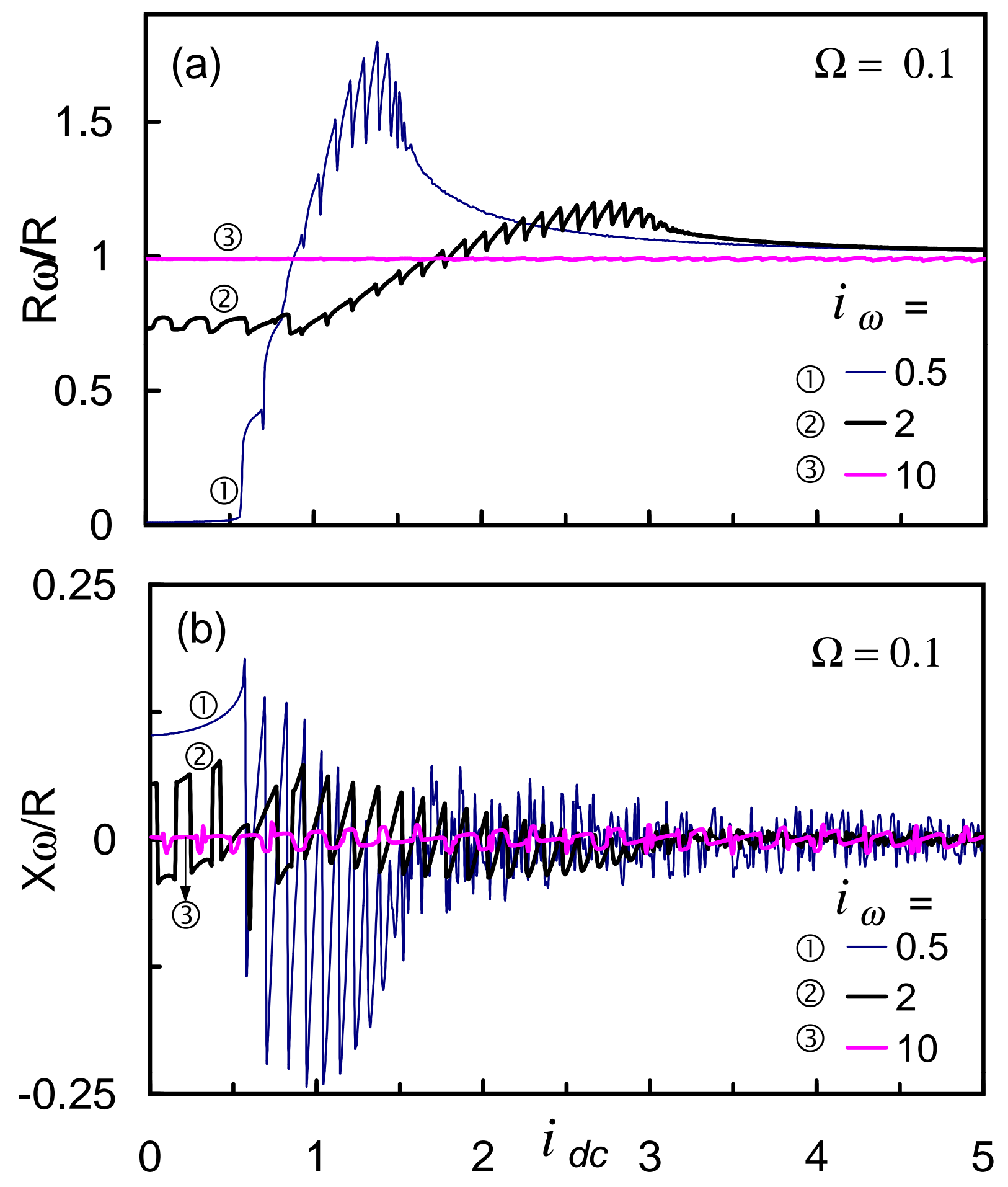


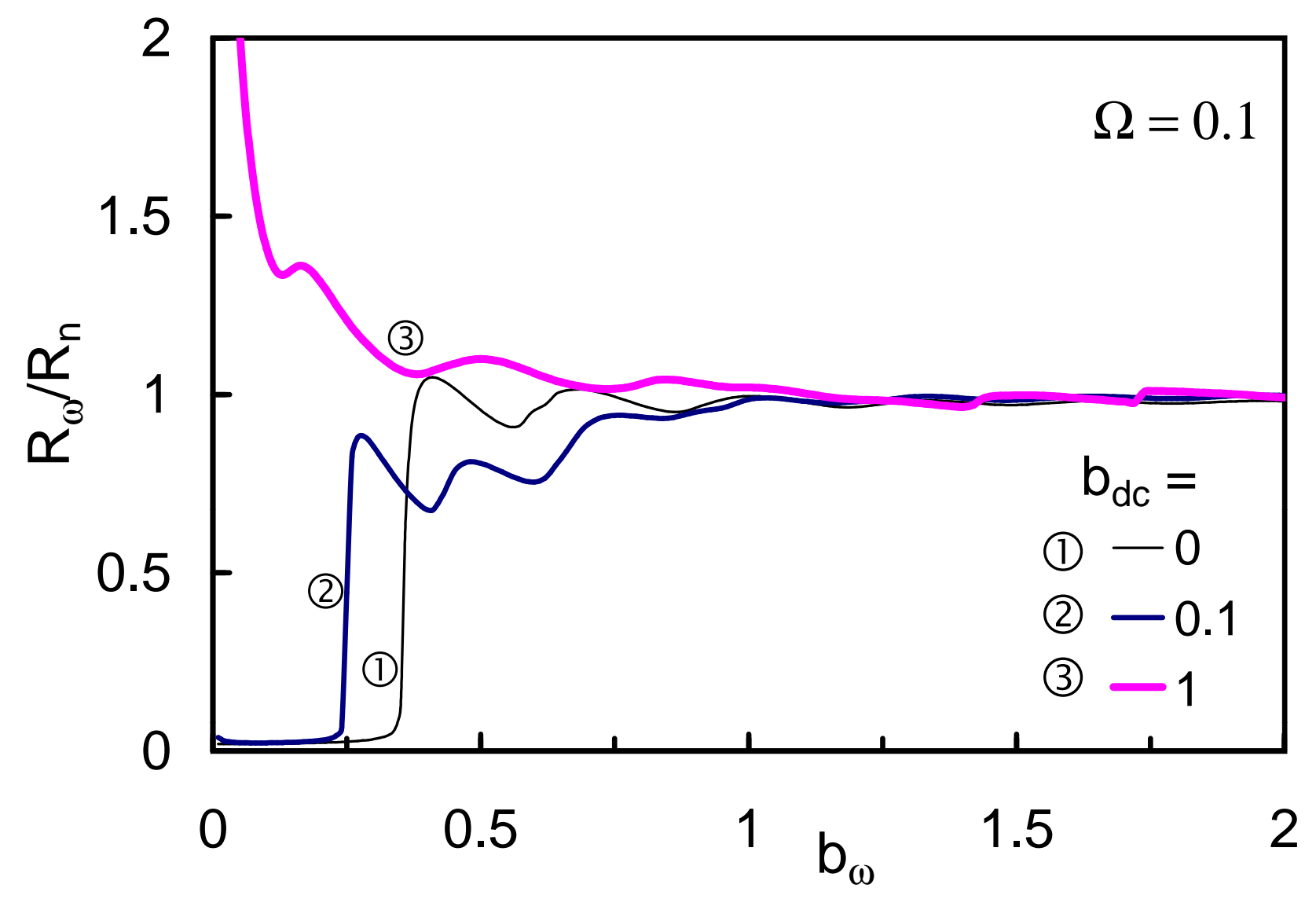




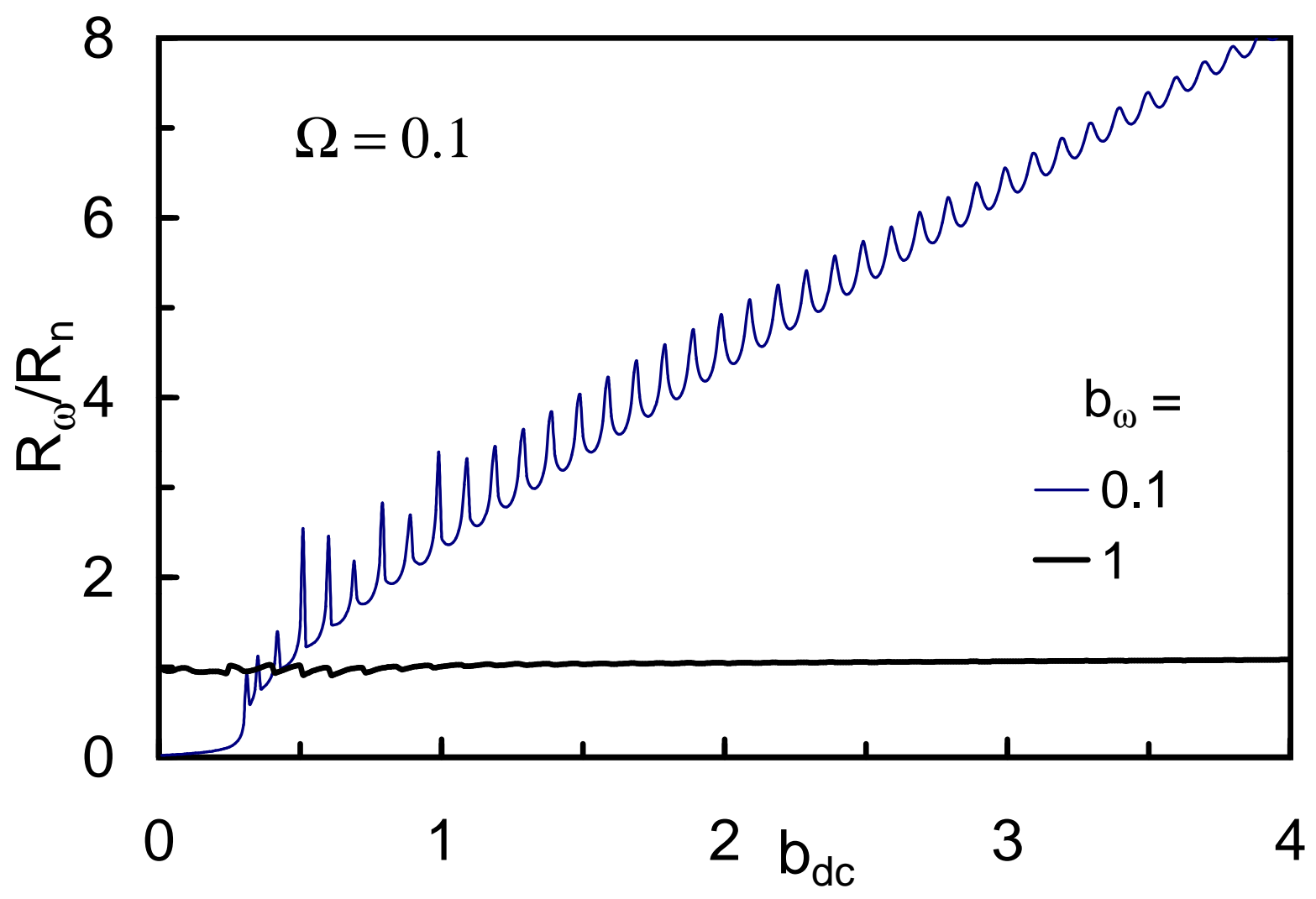




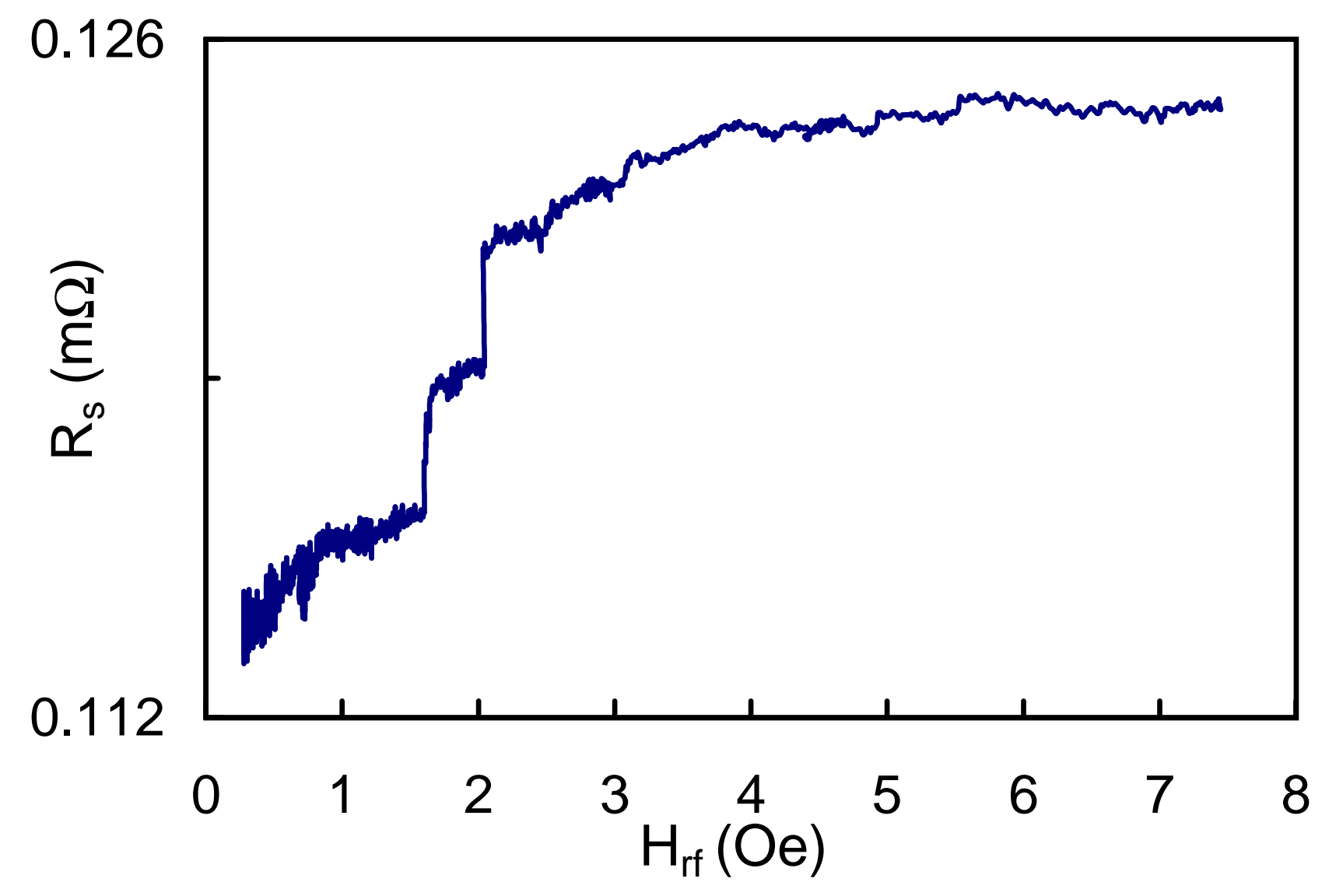

